\title{
Host Specificity of Sporisorium reilianum Is Tightly Linked to Generation of the Phytoalexin Luteolinidin by Sorghum bicolor
}

\author{
Katja Zuther, ${ }^{1,2}$ Jörg Kahnt, ${ }^{2}$ Jan Utermark, ${ }^{1}$ Julia Imkampe, ${ }^{1}$ Simon Uhse, ${ }^{1}$ and Jan Schirawski ${ }^{1,2}$ \\ ${ }^{1}$ Georg-August-Universität Göttingen, Albrecht-von-Haller Institute for Plant Sciences, Julia-Lermontowa-Weg 3, 37077 \\ Göttingen, Germany; ${ }^{2}$ Max Planck Institute for Terrestrial Microbiology, Karl-von-Frisch Str. 10, 35043 Marburg, Germany
}

Submitted 15 December 2011. Accepted 22 May 2012.

\begin{abstract}
The smut fungus Sporisorium reilianum occurs in two varieties (S. reilianum f. sp. reilianum and $S$. reilianum f. sp. zeae) that cause head smut disease on sorghum and maize, respectively. Prior to plant infection, compatible haploid sporidia of $S$. reilianum fuse to form infectious dikaryotic hyphae that penetrate the leaf surface, spread throughout the plant, and reach the inflorescences, in which spore formation occurs. To elucidate the basis of host specificity of the two $S$. reilianum varieties, we compared disease etiology of $S$. reilianum f. sp. reilianum and $S$. reilianum f. sp. zeae on sorghum and maize. Both varieties could penetrate and multiply in both hosts. However, red spots appeared on inoculated leaves after sorghum infection with $S$. reilianum f. sp. zeae. Using matrix-assisted laser desorption-ionization time of flight analysis of leaf extracts, we show that sorghum reacts with the production of the red and orange phytoalexins luteolinidin and apigeninidin upon colonization by $S$. reilianum f. sp. zeae but not by $S$. reilianum f. sp. reilianum. Using in vitro growth assays, we demonstrate that luteolinidin but not apigeninidin slows vegetative growth of both $S$. reilianum f. sp. zeae and $S$. reilianum f. sp. reilianum. However, the phytoalexin biosynthesis gene $S b D F R 3$ is only induced in sorghum after infection with $S$. reilianum f. sp. zeae, as shown by quantitative real-time polymerase chain reaction. This suggests that regulation of luteolinidin biosynthesis determines infection success of $S$. reilianum on sorghum.
\end{abstract}

Many plant pathogenic fungi show a high degree of adaptation to their host plant. This holds especially true for biotrophic fungi that can often infect only a single plant species. How this specificity arises is not known in detail; however, it is likely explained by the ability or inability of the pathogen to overcome the plant's specific defense responses.

The mechanism of host specificity has been elucidated for some necrotrophic fungal plant pathogens. Necrotrophs kill their host plants and live saprophytically on the dead cells. They pro-

Current address for J. Schirawski: RWTH Aachen University, Institute of Applied Microbiology, Department of Microbial Genetics, Biologie 42D 1.152, Worringer Weg 1, 52074 Aachen, Germany.

Corresponding author: J. Schirawski; Telephone: +241-8026616; Fax: +2418022180; E-mail: jan.schirawski@ @wth-aachen.de

* The $\boldsymbol{e}$-Xtra logo stands for "electronic extra" and indicates that one supplementary figure is published online and that Figure 2 appears in color online. duce host-specific toxins that lead to cell death of susceptible plants. Horizontal transfer of genes encoding host-specific toxins can lead to an altered host specificity of the recipient and the emergence of new pathogens for a certain plant species. A famous example is the emergence of tan spot disease on wheat, which seems to be due to lateral gene transfer of the toxA gene of the wheat blotch pathogen Stagonospora nodorum to Pyrenophora tritici-repentis (Friesen et al. 2006, 2008). In Alternaria alternata, host specificity of the different pathotypes has been shown to be dependent on the presence of mobile, conditionally dispensable chromosomes encoding toxin genes that enable its carrier to infect strawberry, apple, tomato, or Japanese pear (Akamatsu et al. 1999; Mehrabi et al. 2011; Tanaka et al. 1999).

Host cell death does not always have a negative consequence for the plant. Targeted killing of specific plant cells is a very potent mechanism of defense against biotrophic plant pathogens that depend on living host cells to survive, colonize, and reproduce. Thus, for biotrophic pathogens, infection success depends, to a high degree, on the ability of the pathogen to suppress the induction of plant defense responses leading to programmed cell death. Even a temporary suppression or a delay of programmed cell death can allow efficient plant colonization, as has been shown for infection of sorghum by the hemibiotroph Colletotrichum sublineolum. Conidia of C. sublineolum show a lower germination and leaf penetration rate on cultivars that quickly respond with $\mathrm{H}_{2} \mathrm{O}_{2}$ accumulation than on those that display a delayed response (Basavaraju et al. 2009). One of the defense responses of sorghum is the generation of phytoalexins, low molecular weight antimicrobial compounds that are de novo biosynthesized upon microbial attack (Nicholson et al. 1987). A successful strategy for microbes to overcome the deleterious effects of phytoalexins is targeted enzymatic detoxification (Pedras and Ahiahonu 2005). However, no mechanisms are known that would detoxify the sorghumspecific 3-deoxyanthoycanidin phytoalexins.

The main 3-deoxyanthocyanidins produced by Sorghum bicolor are luteolinidin and apigeninidin (Lo et al. 1999). Biosynthesis of luteolinidin and apigeninidin has been shown to be initiated by the dihydroflavonol 4-reductase3 of Sorghum bicolor (SbDFR3) that converts flavanones to flavan-4-ols (Liu et al. 2010). Interestingly, resistance and susceptibility of different sorghum cultivars has been associated with the cultivar's ability to synthesize luteolinidin rather than apigeninidin. When challenged with $C$. sublineolum or the nonsorghum pathogen Cochliobolus heterostrophus, the resistant sorghum cultivar SC748-5 produces luteolinidin, methoxy-luteolinidin, apigeninidin, and the caffeic acid ester of arabinosyl 5-O-apigeninidin, while the susceptible sorghum cultivar BTx623 only pro- 
duces apigeninidin and its derivative (Lo et al. 1999). In this study, a sorghum cultivar capable of luteolinidin biosynthesis is challenged with two different varieties of the same fungus, Sporisorium reilianum.

S. reilianum is a biotrophic phytopathogen causing head smut of maize and sorghum. Although syringe infection of seedling plants with fungal cultures is more effective (Prom et al. 2011), the main source of $S$. reilianum infection in the field is via spore-contaminated soil (Matyac and Kommedahl 1985a). Spores of $S$. reilianum germinate to give rise to four haploid sporidia of a different mating type that live as saprobes and multiply by budding (Hanna 1929; Schirawski et al. 2005). Mating of compatible haploid sporidia and the formation of dikaryotic hyphae is a prerequisite for plant infection. Dikaryotic hyphae can penetrate the leaf surface and colonize the host plant initially without causing severe symptoms (Schirawski et al. 2010). Early symptoms observed include the appearance of chlorotic spots on leaves and stems that disappear at later stages (Martinez et al. 2002; Matyac and Kommedahl 1985b; Touraud et al. 1997). In spite of an occasional decrease in plant height (Stromberg et al. 1984), S. reilianum infection does not noticeably affect plant development until the onset of flower formation. Inflorescences from infected plants are partly or completely replaced by fungal sori or form phyllodied panicles in which all floral organs are replaced by leaf-like structures (Ghareeb et al. 2011; Schirawski et al. 2010; Stromberg et al. 1984). Sori are covered by a white peridium that easily breaks to release millions of diploid spores.

$S$. reilianum occurs in several races that belong to two different pathogenically distinct race groups (Halisky 1963), which have been named as the formae speciales $S$. reilianum f. sp. zeae and $S$. reilianum f. sp. reilianum (Martinez et al. 1999). S. reilianum f. sp. zeae is known as a pathogen of maize (Zea mays), while races belonging to $S$. reilianum $\mathrm{f}$. $\mathrm{sp}$. reilianum have been described to infect the closely related host plants sorghum (Sorghum bicolor) and sudan grass (Sorghum sudanense) and,
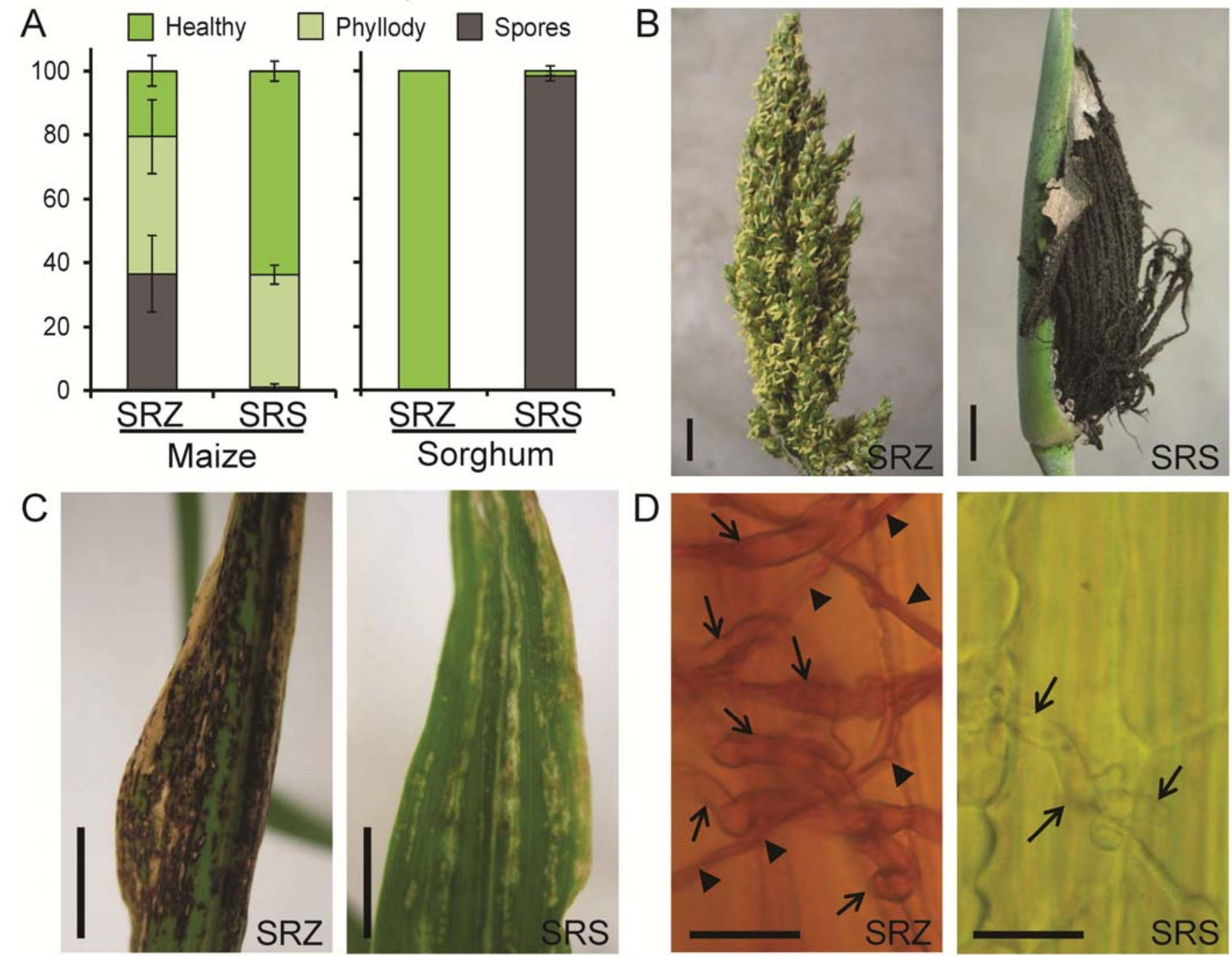

D
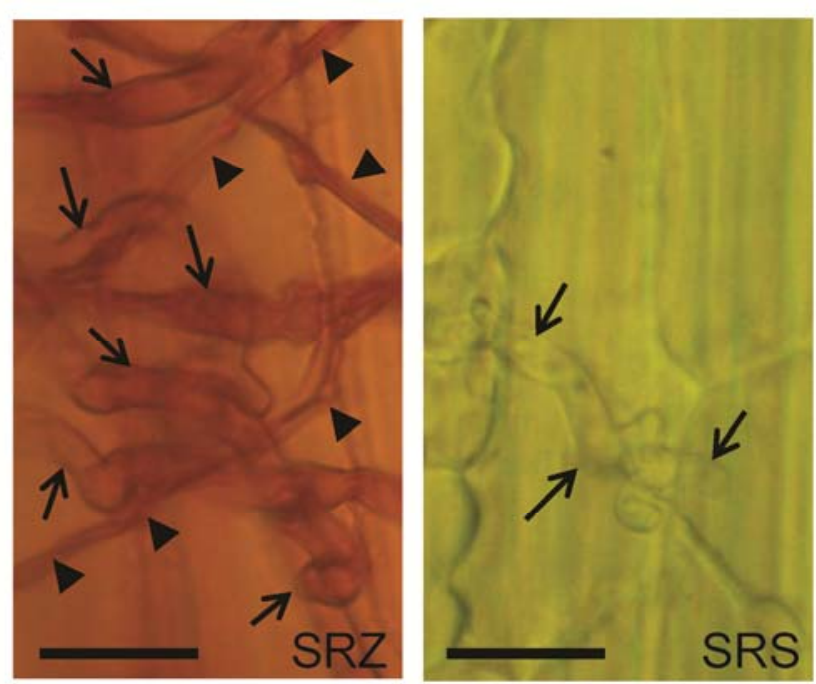

Fig. 1. Symptoms of Sporisorium reilianum infection. A, Virulence of S. reilianum on maize and sorghum. Seedlings of Zea mays 'Gaspe Flint' and Sorghum bicolor 'Tall Polish' were inoculated with a mixture of the compatible haploid S. reilianum strains SRZ1_5-2 and SRZ2_5-1 (SRZ) or SRS1_H2-8 and SRS2_H2-7 (SRS). Symptoms (phyllody or spore formation) were scored in the inflorescences at 7 weeks (maize) or 12 to 16 weeks (sorghum) after inoculation. Data shown are means of three (sorghum) and four (maize) inoculation experiments. For each experiment, 15 to 23 plants were tested. Error bars show standard error of the means. B, Inflorescence symptoms of S. reilianum infection of sorghum. Inoculation of sorghum seedlings with SRZ led to healthy panicles (left), while inoculation with SRS replaced the inflorescence by a fungal sorus containing masses of brown spores (right). C, Leaf symptoms of S. reilianum infection of sorghum. At 7 days after inoculation, sorghum seedlings showed red spots on leaves inoculated with SRZ (left) and mild chlorosis on leaves inoculated with SRS (right). D, Hyphal growth in inoculated leaves. Fungal hyphae (arrows) appeared red in sorghum leaves inoculated with SRZ (left) or colorless when inoculated with SRS (right). Some hyphae appeared collapsed (arrowheads). Pictures were taken at 4 days after inoculation. Size bars: $1 \mathrm{~cm}$ in B and C, $10 \mu \mathrm{m}$ in D. 
occasionally, maize (Al-Sohaily and Mankin 1960; Halisky 1963). It has been suggested that the maize race may have evolved from the sorghum race (Halisky 1963). It is unknown why the maize race is unable to generate spores in sorghum or which molecular differences between the races determine host selection in $S$. reilianum.

Here, we provide an initial explanation for the different colonization behaviors shown by different formae speciales of $S$. reilianum on sorghum. We confirm that $S$. reilianum $\mathrm{f}$. sp. zeae is virulent on maize but not on sorghum and demonstrate that $S$. reilianum f. sp. zeae on sorghum induces the sorghum-specific phytoalexin luteolinidin. We show that luteolinidin slows growth of both $S$. reilianum varieties in vitro. Congruently, luteolinidin biosynthesis is not induced when sorghum is colonized by strains of $S$. reilianum f. sp. reilianum that are fully virulent on sorghum. This suggests that host specificity on sorghum is determined by the ability or inability of the two $S$. reilianum varieties to suppress or induce luteolinidin biosynthesis.

\section{RESULTS}

\section{S. reilianum varieties show host-specific virulence.}

To test whether $S$. reilianum constitutes a suitable model to investigate the molecular basis of host specificity, we first verified that maize or sorghum, respectively, are host plants for the two different formae speciales of $S$. reilianum. To this end, we isolated compatible haploid strains from single spores of spore samples collected from infected maize or infected sorghum. The strains isolated from the spore sample of infected maize were called SRZ1_5-2 (mating type alb1) and SRZ2_5-1 (mating type $a 2 b 2$ ) and were described previously (Schirawski et al. 2005). The strains isolated from the spore sample of infected sorghum were called SRS1_H2-8 (mating type alb1) and SRS2_H2-7 (mating type $a 2 b 6$ ).

We either used a mixture of the compatible haploid strains SRZ1_5-2 and SRZ2_5-1 (hereafter referred to as SRZ) or a mixture of the compatible haploid strains SRS1_H2-8 and SRS2_H2-7 (hereafter referred to as SRS) to inoculate 7-dayold seedlings of Z. mays 'Gaspe Flint' and 14-day-old seedlings of Sorghum bicolor 'Tall Polish'. Disease symptoms were scored at flowering time. Inoculation of maize with SRZ resulted in about $36 \%$ of the plants showing spores with an additional $43 \%$ showing phyllody in inflorescences (Fig. 1A, left). In contrast, SRZ did not lead to any spore formation on infected sorghum plants (Fig. 1A and B, right and left panel, respectively). Infection of sorghum with SRS resulted in $98 \%$ of the plants with spores (Fig. 1A and B, right panels). When SRS was used to inoculate maize, no spore formation was observed. However, a fraction of the plants (35\%) showed formation of phyllody (Fig. 1A, left), indicating that SRS can indeed colonize maize and cause symptoms in female inflorescences. This shows that the two different $S$. reilianum varieties, $S$. reilianum f. sp. reilianum and $S$. reilianum f. sp. zeae, have different host specificities. While SRZ produce spores only on maize, SRS prefers sorghum as host for spore formation.

\section{Infection of sorghum with SRZ leads to formation of red spots.}

At 7 days after inoculation, mild chlorosis could be observed on sorghum leaves inoculated with SRS (Fig. 1C, right). In contrast, leaves inoculated with SRZ developed dark red spots (Fig. $1 \mathrm{C}$, left). These symptoms became more severe with time and, at 15 days after inoculation, SRZ-inoculated leaves were completely scattered with dark lesions (not shown). To assess whether the appearance of red spots is correlated with the presence of fungal hyphae, affected leaves were subjected to light microscopy. Hyphae of SRZ were clearly visible within the red lesions (Fig. 1D, left). In and near the red spots, SRZ hyphae were stained in red. In contrast, hyphae of SRS did not show any particular stain (Fig. 1D, right). This suggests that one or several red compounds are produced in sorghum leaves as a specific response to infection with SRZ, which does not occur after infection with SRS. In addition, hyphal staining indicates either a localized deposition of the red material or a high affinity of one or more red compounds towards fungal cell wall components.

\section{Red spots contain the phytoalexin luteolinidin.}

To determine the nature of the red compounds, plant leaf extracts from sorghum plants infected either with SRS or SRZ were analyzed by Nano-Liquid Chromatography. At $480 \mathrm{~nm}$, several peaks were clearly visible in extracts from SRZ-infected leaf material but not in those from SRS-infected leaf material (Fig 2A, lower panel). Since sorghum is known to react to pathogen attack with the formation of the sorghum-specific phytoalexins apigeninidin and luteolinidin (Nicholson et al. 1987), we compared the elution profiles of the extracts with those of the commercial phytoalexins. The main peaks in the elution profile of the extracts of SRZ-infected sorghum leaves eluted at 43 and $46 \mathrm{~min}$, respectively, which corresponded to the elution times of luteolinidin (43 min) and apigeninidin (46 min) (Fig. 2A, upper panel).

To identify whether luteolinidin and apigeninidin were present in extracts of SRZ-infected leaves, eluting compounds were collected and subjected to matrix-assisted laser desorption-ionization time of flight (MALDI-TOF) analysis. The spectrum resulted in several molecule peaks. One molecule peak had a mass-to-charge ratio of 255.03 that corresponded to the calculated mass of apigeninidin (255.07), and one had a mass-to-charge ratio of 271.03 that corresponded to the calculated mass of luteolinidin (271.06; Fig. 2B). To determine the exact identity of one or more compounds, the mass peaks with the highest signal-to-noise ratio at 255 and 271 were subjected to fragmentation. The fragmentation pattern of the compound present in leaf extracts with the mass-to-charge ratio of 255 was very similar to that of fragmented apigeninidin (Fig. 2C), and the fragmentation pattern of the compound with the massto-charge ratio of 271 was very similar to that of luteolinidin standard (Fig. 2D). Both compounds were also identified as main components when the red spots that appeared on sorghum leaves after inoculation with SRZ were excised from the inoculated leaf before extract preparation to remove most of the green material (data not shown). This confirms the presence of both apigeninidin and luteolinidin in sorghum leaves inoculated with SRZ as the major 3'-deoxyanthocyanidins present. None of these compounds were detected in leaves inoculated with SRS (Fig. 2A; data not shown).

\section{Phytoalexin biosynthesis genes are up-regulated in SRZ-infected sorghum leaves.}

To assess whether 3'-deoxyanthocyanidin accumulation is caused by an increased rate in phytoalexin biosynthesis, we determined expression of the 3'-deoxyanthocyanidin biosynthetic enzyme dihydroflavonol 4-reductase encoded by SbDFR3 (Liu et al. 2010). We isolated total RNA from leaf tissues of sorghum plants five days after inoculation with water, SRZ, or $\mathrm{SRS}$, and determined expression of $S b D F R 3$ relative to the sorghum actin gene SbActin by quantitative real-time polymerase chain reaction (RT-PCR). Plants inoculated with SRS showed the same level of $S b D F R 3$ expression as mock-inoculated plants (Fig. 3). In contrast, $S b D F R 3$ was strongly up-regulated in leaf samples inoculated with SRZ (Fig. 3). This indicates that colonization of sorghum by SRZ induces expression of biosynthetic enzymes essential for phytoalexin generation. 




B

C
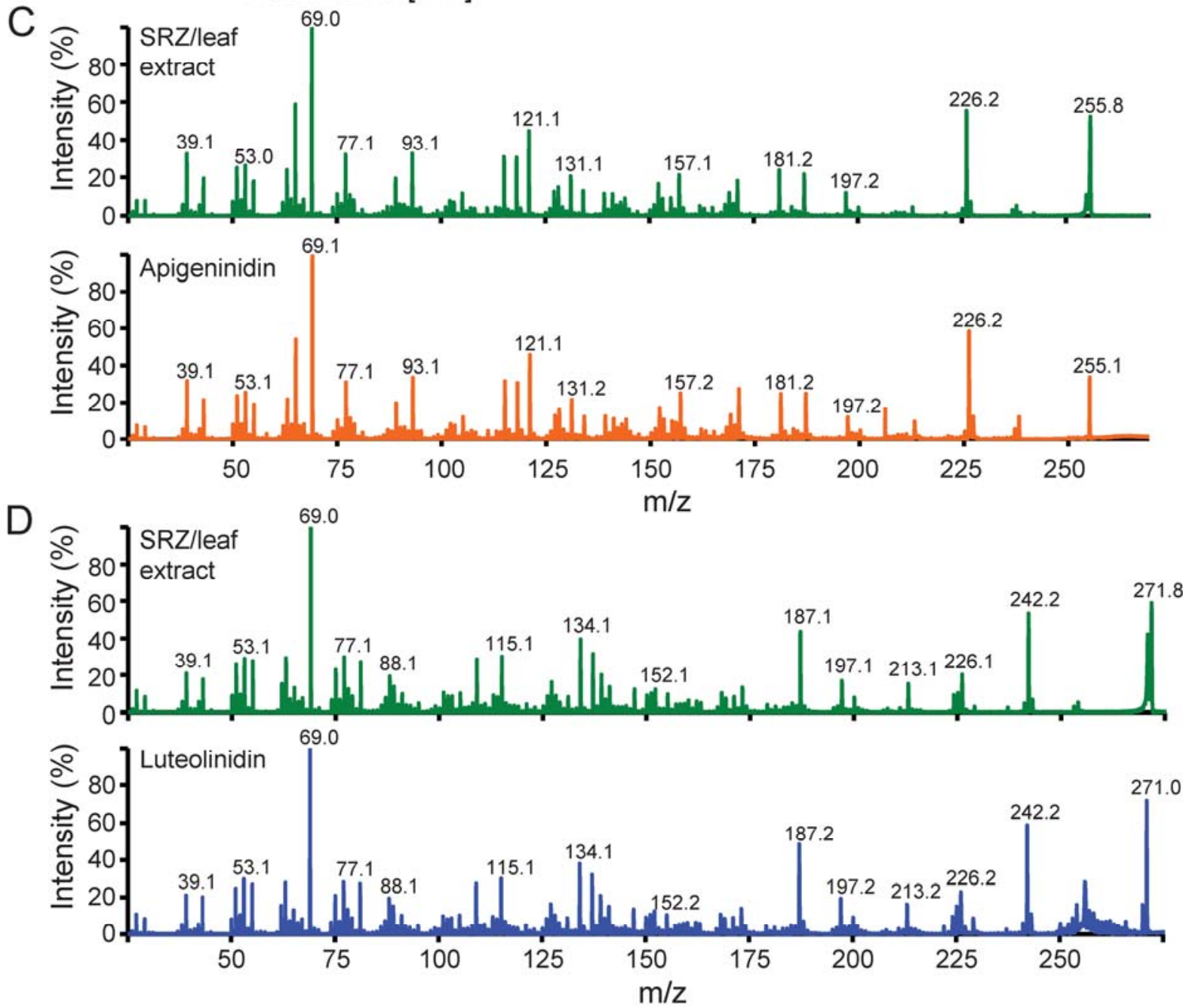

Fig. 2. Identification of phytoalexins in extracts of Sporisorium reilianum-infected sorghum leaves. A, Nano liquid chromatography profiles of luteolinidin and apigeninidin and of extracts of sorghum leaves at 15 days after inoculation with a mixture of the compatible S. reilianum f. sp. zeae strains SRZ1_5-2 and SRZ2_5-1 (SRZ) or a mixture of the compatible S. reilianum f. sp. reilianum strains SRS1_H2-8 and SRS2_H2-7 (SRS). Phytoalexins were extracted from inoculated leaves by soaking in technical ethanol for $72 \mathrm{~h}$. B, Matrix-assisted laser desorption-ionization time of flight analysis of substances in the extract of SRZ-inoculated leaves. Molecule peaks with mass-to-charge ratios of 271.03 and 255.03 could be detected. C, Comparison of the fragmentation patterns of the substance with the mass-to-charge ratio of 255 in the extract of SRZ-inoculated leaves (top) with that of apigeninidine (bottom). D, Comparison of the fragmentation patterns of the substance with the mass-to-charge ratio of 271 in the extract of SRZ-inoculated leaves (top) with that of luteolinidine (bottom). 


\section{Luteolinidin but not apigeninidin slows growth of $S$. reilianum.}

To test whether the presence of 3'-deoxyanthocyanidins in SRZ-infected sorghum leaves could be a possible reason for the absence of spore formation by SRZ on sorghum, we investigated whether luteolinidin affects the proliferation capability of $S$. reilianum in vitro. Strains of both varieties were grown in liquid medium to mid-exponential phase, and equal aliquots of the cultures were dispersed evenly on individual agar plates lacking or containing luteolinidin $(1.5 \mathrm{mM}, 3 \mathrm{mM})$, apigeninindin $(1.5 \mathrm{mM}, 3 \mathrm{mM})$, or a mixture of luteolinidin and apigeninidin ( $3 \mathrm{mM}$ each). At four days after plating, all strains grew as well on apigeninidin as on plates without the phytoalexin (Supplementary Fig. S1; data not shown). On plates containing luteolinidin, colonies appeared smaller than on those lacking the phytoalexin, and a higher luteolinidin concentration had a stronger effect (Fig. 4A; data not shown). In addition to the strains SRZ1_5-2 and SRZ2_5-1, also the sorghum infecting strains SRS1_H2-8 and SRS2_H2-7 showed growth inhibition in the presence of luteolinidin (Fig. 4A), This growth inhibition was not increased, if the plates contained in addition $3 \mathrm{mM}$ apigeninidin. This indicates that luteolinidin but not apigeninidin negatively affects the proliferation capability of both $S$. reilianum varieties.

To determine whether growth rate of the $S$. reilianum strains was affected in the presence of luteolinidin, we measured the average colony diameter of ten individual colonies at seven successive days using SRZ1_5-2 and calculated colony areas. The colony area expanded more slowly when the colonies were growing in the presence of luteolinidin than without the



Fig. 3. Quantification of $S b D F R 3$ gene expression. SbDFR3 gene expression was assessed by real-time polymerase chain reaction on RNA samples extracted from inoculated sorghum seedlings at 5 days after inoculation with water (Mock), a mixture of the compatible Sporisorium reilianum f. sp. reilianum strains SRS1_H2-8 and SRS2_H2-7 (SRS), or a mixture of the $S$. reilianum $\mathrm{f}$. sp. zeae strains SRZ1_5-2 and SRZ2_5-1 (SRZ). Expression of $S b D F R 3$ relative to that of sorghum actin is highly induced upon exposure to SRZ. Error bars show standard error of the means of three biological replicates. phytoalexin (Fig. 4B and C). This shows that luteolinidin slows growth of $S$. reilianum in vitro.

\section{DISCUSSION}

Strains of $S$. reilianum f. sp. reilianum are very efficient sorghum pathogens and rarely succeed to cause smut disease on
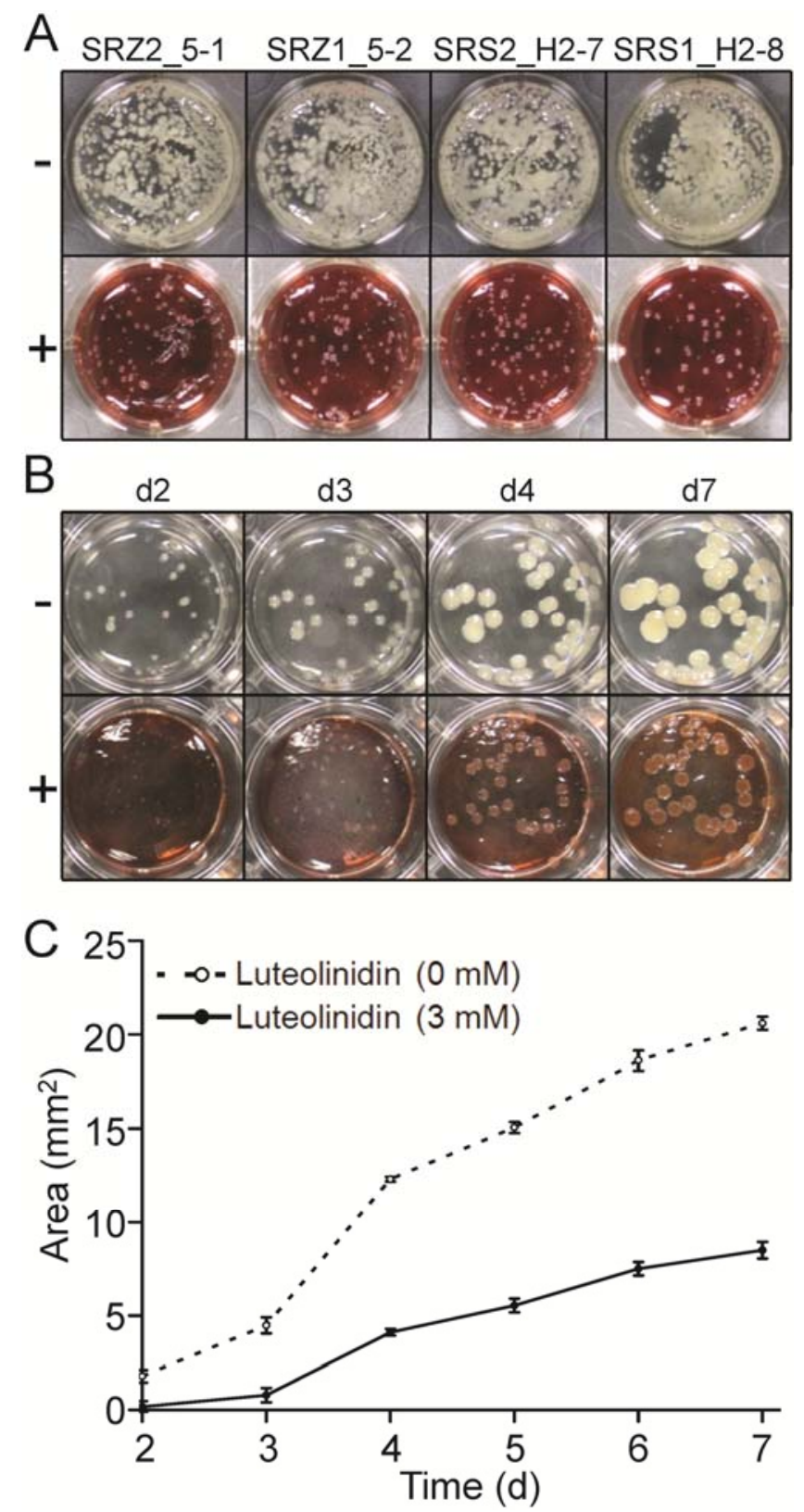

Fig. 4. Impact of luteolinidin on growth of Sporisorium reilianum. A, Luteolinidin similarly affects growth of $S$. reilianum strains of both varieties. The strains indicated at the top were pregrown in YEPSlight $(10 \mathrm{~g}$ of yeast extract, $4 \mathrm{~g}$ of Bacto peptone, and $4 \mathrm{~g}$ of sucrose per liter) liquid culture and were plated on regeneration agar containing $3 \mathrm{mM}$ luteolinidin (bottom) or only the solvent (top). The presence of luteolinidin led to smaller colonies after 4 days of incubation at $28^{\circ} \mathrm{C}$. B, The presence of luteolinidin slows growth of $S$. reilianum. The $S$. reilianum $\mathrm{f}$. $\mathrm{sp}$. zeae SRZ1_5-2 was pregrown in YEPSlight liquid culture and was plated on regeneration agar containing $3 \mathrm{mM}$ luteolinidin (bottom) or only the solvent (top). Pictures of plates were taken on seven successive days. Colonies grow more slowly in the presence of luteolinidin. C, Quantification of the data illustrated in B. Diameters of 10 colonies of SRZ1_5-2 were measured and averaged and were used to calculate colony areas. Data shows averages and standard error of the means of three independent experiments. 
maize. In contrast, strains of $S$. reilianum f. sp. zeae can form spores on maize but are unable to do so on sorghum. We could show that the maize-infecting strains colonize sorghum leaves, on which they induce the formation of red spots containing the 3'-deoxyanthocyanidins luteolinidin (which displays a deep red color in solution) and apigeninidin (which solves to a light orange). Of these, at least luteolinidin behaves like a phytoalexin against SRZ. Luteolinidin hampers vegetative growth of $S$. reilianum, its biosynthesis gene $S b D F R 3$ is highly up-regulated in the presence of SRZ, indicating de novo biosynthesis, and the red phytoalexin accumulates to high concentrations around fungal hyphae, as judged by microscopic analysis. Thus, luteolinidin generation seems to be a specific, induced, and targeted plant defense response.

Previously, other in vitro assays were used to measure fungitoxicity of sorghum-specific phytoalexins that included monitoring spore germination, germ-tube elongation, and germ-tube abnormalities of Colletotrichum graminicola and Helminthosporium maydis (Nicholson et al. 1987). An effect of luteolinidin on germ-tube length was shown for $C$. graminicola and on germ-tube bifurcation for $H$. maydis. However, no effect was seen on spore germination of $C$. graminicola (Nicholson et al. 1987). Possibly, a compound concentration well below the value occurring in planta was used. Growth of $S$. reilianum was affected in the presence of $3 \mathrm{mM}$ luteolinidin. This concentration equals the measured level of luteolinidin accumulation in sorghum leaves $72 \mathrm{~h}$ after inoculation with Cochliobolus heterostrophus (Shih et al. 2007). Since the phytoalexins are delivered to the site of fungal penetration with the help of vesicles that fuse with the cell membrane (Snyder and Nicholson 1990), it is possible that fungal hyphae are exposed locally to much higher phytoalexin concentrations.

While a rapid autodestruction of host cells encountering attempted penetration events is an efficient plant defense strategy towards biotrophic pathogens (Greenberg 2007), host cell killing through the phytotoxic effects of the phytoalexins does not seem to be the strategy employed for preventing entry of SRZ in sorghum leaves. Red spots appeared on inoculated leaves at 4 to 7 days postinoculation. Since plant penetration occurs within the first $24 \mathrm{~h}$ after inoculation (Schirawski et al. 2010), the late appearance of red spots indicates that accumulation of phytoalexins is not used to prevent fungal leaf penetration. In contrast, the phytoalexins could serve to slow growth and proliferation of the fungus during plant colonization. Since the fungus causes symptoms only in sorghum inflorescences that emerge at the very top of the plant, a delay in proliferation causing the fungus not to reach the inflorescence in time, might have a severe impact on the success rate of spore formation.

Pathogens that overcome resistance caused by the generation of toxins and phytoalexins by the plant often have very efficient detoxification mechanisms (VanEtten et al. 1989). In vitro experiments showed that vegetative growth of SRZ1_5-2, SRZ2_5-1, SRS1_H2-8, and SRS2_H2-7 was similarly disturbed by the presence of luteolinidin in the growth medium. This suggests that SRS1_H2-8 and SRS2_H2-7 are not more resistant against the toxic effects of luteolinidin than are SRZ1_5-2 and SRZ2_5-1. Rather, it could be shown (Fig. 3) that phytoalexin biosynthesis is not induced upon sorghum inoculation with a mixture of SRS1_H2-8 and SRS2_H2-7. Thus, $S$. reilianum f. sp. reilianum does not resort to degradation of phytoalexins as infection strategy. Instead, it either escapes detection by the plant and does not elicit the generation of phytoalexins or it has very efficient means to suppress phytoalexin biosynthesis (discussed below).

Infection of sorghum with SRS does not lead to luteolinidin or apigeninidin generation, although the compounds are read- ily produced upon infection with SRZ. It is possible that SRZ generates or carries elicitors that trigger phytoalexin biosynthesis and that are either not generated or not exposed by SRS. Phytoalexin accumulation has been observed to occur in sorghum even before plant penetration by hyphae of $C$. graminicola or C. sublineolum (Du et al. 2010; Liu et al. 2010), suggesting a pathogen-associated molecular pattern-elicited response. If the response was triggered by perception of conserved molecules emanating from the fungal surface or from plant degradation products, phytoalexins should also be generated in response to infection by SRS, unless SRS actively suppresses phytoalexin biosythesis or disguises its elicitors to escape recognition. If $S$. reilianum $\mathrm{f}$. sp. reilianum actively and efficiently suppresses phytoalexin induction, then suppression does not seem to occur in systemic cells. Coinfection of both varieties led to the same strong phytoalexin generation as in the control infection with SRZ alone (not shown; discussed below).

When sorghum was infected with the hemibiotroph C. graminicola, a strong phytoalexin response occurred within the first $45 \mathrm{~h}$, effectively blocking fungal entry (Snyder and Nicholson 1990). The relatively delayed response observed after exposure to SRZ might be due to the use of a different sorghum cultivar. Different sorghum cultivars differ in phytoalexin composition, amount, and speed of phytoalexin generation (Du et al. 2010; Liu et al 2010). The cultivar SC784-5, which is resistant to $C$. sublineolum, has been shown to accumulate the flavone luteolin much earlier and in higher concentrations than the susceptible cultivar BTx623, which produces both luteolin and apigenin (Du et al. 2010). However, exposure of sorghum cultivar Tall Polish to conidia of $C$. graminicola led to rapid $(<48 \mathrm{~h})$ phytoalexin generation (not shown, discussed below). Alternatively, the relatively delayed response may be due to initial suppression of phytoalexin biosynthesis that is sustained in the case of $S$. reilianum $\mathrm{f}$. sp. reilianum but can be overcome in the case of $S$. reilianum $\mathrm{f}$. $\mathrm{sp}$. zeae or be due to a different capacity of both varieties to disguise their elicitor molecules, possibly by fungal effector proteins that are only expressed after leaf colonization and have different primary sequences in both varieties.

Weakly conserved fungal effector proteins have been identified when comparing the genomes of $S$. reilianum and of the close relative Ustilago maydis, the causal agent of maize smut disease. Many of these weakly conserved proteins were encoded in gene loci, and some of these loci have been proven to affect spread of $U$. maydis through the plant, either negatively or positively (Schirawski et al. 2010).

It will be very interesting to analyze the genomic differences between $S$. reilianum f. sp. zeae and $S$. reilianum f. sp. reilianum to uncover the molecular basis of host colonization and the different mechanisms employed by these closely related $S$. reilianum varieties. The presence of factors that allow or prevent colonization of a certain host may be at the origin of the evolution of new smut fungal species by reproductive isolation.

\section{MATERIALS AND METHODS}

\section{Strains, plant material, and growth conditions.}

Compatible haploid strains of $S$. reilianum were isolated from individual spores of spore samples found on maize in Hohenheim, Germany (SRZ1_5-2 [albl] and SRZ2_5-1 [a2b2], described previously [Schirawski et al. 2005]) or on sorghum in Corpus Christi, Texas, U.S.A. (provided by G. Odvody). To isolate compatible haploid strains from single spores, individual spores were placed on a potato dextrose (PD) agar plate using a micromanipulator. Colonies resulting from spore germination were streaked for individual colonies on fresh PD plates. Individual colonies from these plates were 
checked for mating ability with tester strains (Schirawski et al. 2005) and two compatible strains (SRS1_H2-8 [alb1] and SRS2_H2-7 [a2b6]) were selected. S. reilianum strains were stocked at $-80^{\circ} \mathrm{C}$ in NSY-Glyc containing nutrient broth (Becton, Dickinson and Company [BD], Heidelberg, Germany; $8 \mathrm{~g}$ per liter), yeast extract (BD, 1 g per liter), sucrose (Roth, Karlsruhe, Germany; 5 g per liter), and glycerol (Roth; 70\%).

Strains were grown from stock on PD plates at $28^{\circ} \mathrm{C}$. Colonies were inoculated in $3 \mathrm{ml}$ of YEPSlight (10 $\mathrm{g}$ of yeast extract, $4 \mathrm{~g}$ of Bacto peptone, and $4 \mathrm{~g}$ of sucrose per liter), were cultivated at $28^{\circ} \mathrm{C}$ with shaking for at least $6 \mathrm{~h}$, and were used to inoculate PD broth (BD; $50 \mathrm{ml})$. Cultures were incubated overnight to an optical density at $600 \mathrm{~nm}\left(\mathrm{OD}_{600}\right)$ of 0.5 to 0.8 . Cells were pelleted by centrifugation, were resuspended to a calculated $\mathrm{OD}_{600}$ of 2.0, and were mixed in a 1:1 ratio with the compatible strain immediately prior to plant inoculation. Plant inoculation was done by syringe-assisted deposition of sporidial culture mixtures in the leaf whorls of 7-day-old seedling plants of maize (Zea mays 'Gaspe Flint', provided by R. Kahmann) or 14-day-old seedlings of sorghum (Sorghum bicolor 'Tall Polish', Leibniz Institute of Plant Genetics and Crop Plant Research, Gatersleben). Plants were grown under greenhouse conditions at $28^{\circ} \mathrm{C}$ and $16 \mathrm{~h}$ of illumination and $22^{\circ} \mathrm{C}$ during the 8-h night period. Disease was assessed 7 to 15 days after inoculation for evaluation of phytoalexin production on sorghum or after inflorescence emergence at 7 weeks after inoculation for maize and at 12 to 16 weeks after inoculation for sorghum. For coinfection of both varieties, a mixture of the compatible strains SRS1_H2-8 and SRS2_H2-7 was mixed with the solopathogenic strain $S$. reilianum f. sp. zeae JS161 (Schirawski et al. 2010) in a 10:1 (S. reilianum f. sp. reilianum to $S$. reilianum f. sp. zeae) ratio immediately before plant inoculation. The solopathogenic strain does not undergo a mating reaction and forms infection structures without a mating partner, which prevents hybrid formation between varieties. Control inoculations were done with the mixture of compatible SRS1_H2-8 and SRS2_H2-7 strains mixed with water and with the JS161 strain mixed with water. Phytoalexin formation on leaves was visually evaluated at 6 days postinoculation.

For inoculation of sorghum with $C$. graminicola, leaves of 14-day-old sorghum seedlings were detached and were placed in a humid chamber. Spores of C. graminicola 112 were harvested from 14-day-old plates, were resuspended in $0.01 \%$ (vol/vol) Tween 20, and were used to spray-inoculate sorghum leaves at a concentration of $6 \times 10^{5}$ spores per milliliter. At $42 \mathrm{~h}$ after inoculation, red spots were readily visible, indicating phytoalexin generation.

\section{Quantitative (q)RT-PCR.}

For total RNA extraction, infected leaf material was taken from the inoculation site and was frozen directly in liquid nitrogen. The frozen material $(100 \mathrm{mg})$ was treated with $5 \mathrm{~mm}$ metal beads in the Tissue Lyser LT (Qiagen, Hilden, Germany), according to the instructions of the RNeasy plant mini kit (Qiagen), including on-column treatment with RNase-free DNaseI. Isolated RNA $(1 \mu \mathrm{g})$ was used as a template for firststrand cDNA synthesis with the quantitect reverse transcription kit (Qiagen), following the manufacturer's instructions.

For qRT-PCR analysis, cDNA was diluted 1:10 with water. The reactions were done in triplicate on the CFX96 Real-Time PCR detection system (Bio-Rad, Munich) in a total volume of $20 \mu \mathrm{l}$ containing $1 \mu \mathrm{l}$ of cDNA, $1 \mu \mathrm{l}$ of each primer $(4 \mu \mathrm{M})$, and $10 \mu \mathrm{l} 2 \times$ Sso Fast EvaGreen Supermix (Bio-Rad). Primers used for amplification of SbDFR3 were CL625 and CL26 (Liu et al. 2010) and, for the sorghum actin SbActin that was used as reference, HEL794 and HEL795 (Du et al. 2010). Primers used for amplification of SbDFR3 and SbActin did not lead to any amplification products with cDNA of fungal cultures. Conditions for qRT-PCR amplification were as follows: $95^{\circ} \mathrm{C}$ for $30 \mathrm{~s}, 45$ cycles of $95^{\circ} \mathrm{C}$ for $5 \mathrm{~s}$, and $55^{\circ} \mathrm{C}$ for $10 \mathrm{~s}$. Gene expression and PCR efficiency were calculated using the CFX Manager (Bio-Rad).

\section{Luteolinidin and apigeninidin analysis.}

MALDI-TOF (tandem mass spectrometry [MS/MS]) has been chosen for discrimination of the compounds because of overlapping absorption spectra and similar retention properties of the 3' deoxyanthocyanidins (Lo and Nicholson 1998; Shih et al. 2007).

The samples were extracted with ethanol and were then concentrated under vacuum but not to dryness. The resulting aqueous solution was injected onto a PepMap100 C-18 RP nanocolumn and was separated on an UltiMate 3000 liquid chromatography system (Dionex, Idstein, Germany) in a gradient of increasing concentrations of buffer B $(0.04 \%$ trifluoroacetic acid in a solution of $80 \%$ acetonitrile and $20 \%$ water) in buffer $\mathrm{A}(0.05 \%$ trifluoroacetic acid in water): 0 to $5 \mathrm{~min}$, buffer $\mathrm{A} ; 5$ to $10 \mathrm{~min}$, increase buffer B to $20 \%, 10$ to $50 \mathrm{~min}$, increase buffer B by $1 \%$ per min to $60 \%$; 50 to $60 \mathrm{~min}$, increase buffer B to $100 \%$, and 60 to $70 \mathrm{~min}$, buffer B. Peptides were eluted at a flow rate of $300 \mathrm{nl} / \mathrm{min}$ and were detected at 480 $\mathrm{nm}$. A Probot microfraction collector (Dionex) was used to spot liquid chromatography-separated peptides on a MALDI target with a rate of $8 \mathrm{~s}$ per spot. The eluate was mixed with matrix consisting of $4 \mathrm{mg}$ of $\alpha$-cyano-4-hydroxycinnamic acid per milliliter in $70 \%$ acetonitrile and $0.05 \%$ trifluoroacetic acid.

MALDI-TOF-TOF analysis was carried out on a 4800 plus MALDI TOF/TOF analyzer (Applied Biosystems/MDS Sciex, Foster City, CA, U.S.A.), using the 4800 Series Explorer software. The mass spectrometer was operated in positive-ion reflector mode in a mass range from 0.2 to $2 \mathrm{kDa}$. For one main spectrum, 10 subspectra with 90 spots per subspectrum were averaged. Close external calibration was performed with the calibration standard from BRUKER Daltonics (Bremen, Germany). Internal calibration in every sample spot was automatically performed using the matrix peak at $379.093 \mathrm{kDa}$. In MS/MS positive ion mode, 3,600 spectra were averaged; in collision-induced decay, collision energy was $1 \mathrm{kV}$, collision gas was air, and external calibration was set using the fragments of Glu1-Fibrino-peptide B. Luteolinidin and apigeninidin were detected by comparison of the fragmentation pattern of molecule peaks at the best signal-to-noise ratio with authentic samples that were obtained from Roth and Sigma-Aldrich (Schnelldorf, Germany), respectively.

\section{Phytoalexin toxicity test.}

For toxicity analysis of the phytoalexins luteolinidin and apigeninidin on growth capability of $S$. reilianum, a $6-\mathrm{mM}$ solution of luteolinidin or apigeninidin was generated by first resolving the total amount of phytoalexin in dimethyl sulfoxide (DMSO) before adding water containing 6\% Tween 80 to give a final DMSO concentration of $0.8 \%$. Addition of DMSO and Tween 80 were required for proper solubilization and were, therefore, also included at the same concentration in the mock control. After filter sterilization, the solutions were used to fill prewarmed test tubes. To each test tube, one volume of regeneration agar ( $10 \mathrm{~g}$ of yeast extract, $20 \mathrm{~g}$ of pepton, and $20 \mathrm{~g}$ of sucrose per liter, $1 \mathrm{M}$ sorbitol; $15 \mathrm{~g}$ of agar per liter) was added. The content of the test tube was mixed and was then quickly poured into one well of a six-well plate. $S$. reilianum strains were grown overnight from single colonies in YEPSlight at $28^{\circ} \mathrm{C}$ with shaking. The cultures were diluted $1: 1,000$ in water. From this dilution, $1 \mathrm{ml}$ was evenly distributed on top of the agar in the wells. The plates were incubated 
at $28^{\circ} \mathrm{C}$ for 7 days. At day 2, 3, 4, 5, 6, and 7, the plates were photographed and magnified pictures were used to measure colony diameters that were used to calculate colony areas.

\section{ACKNOWLEDGMENTS}

We thank G. Odvody for supplying spores of S. reilianum on sorghum, S. Wirsel for supplying C. graminicola, M. Wagenknecht for help in isolating SRS1_H2-8 and SRS2_H2-7 from a single spore, T. Wollenberg for variety coinfection experiments, A. Poloni for sorghum inoculation with $C$. graminicola, R. Kahmann for generous support, E. Meyer and J. Krüger for technical assistance, and B. Winterberg for critical comments on the manuscript. This work was supported by the German Initiative of Excellence (Deutsche Forschungsgemeinschaft grant no. ZUK45/1 to J. Schirawski)

\section{LITERATURE CITED}

Akamatsu, H., Taga, M., Kodama, M., Johnson, R., Otani, H., and Kohmoto, K. 1999. Molecular karyotypes for Alternaria plant pathogens known to produce host-specific toxins. Curr. Genet. 35:647-656.

Al-Sohaily, I.A., and Mankin, C. J. 1960. Reaction of corn and sorghum to corn and sudan grass head smuts. Plant Dis. Rep. 44:113-114.

Basavaraju, P., Shetty, N. P., Shetty, H. S., de Neergaard, E., and Jørgensen, H. J. L. 2009. Infection biology and defence responses in sorghum against Colletotrichum sublineolum. J. Appl. Microbiol. 107:404-415.

Du, Y., Chu, H., Wang, M., Chu, I. K., and Lo, C. 2010. Identification of flavone phytoalexins and a pathogen-inducible flavone synthase II gene (SbFNSII) in sorghum. J. Exp. Bot. 61:983-994.

Friesen, T. L., Stukenbrock, E. H., Liu, Z. H., Meinhardt, S., Ling, H., Faris, J. D., Rasmussen, J. B., Solomon, P. S., McDonald, B. A., and Oliver, R. P. 2006. Emergence of a new disease as a result of interspecific virulence gene transfer. Nat. Genet. 38:953-956.

Friesen, T. L., Faris, J. D., Solomon, P. S., and Oliver, R. P. 2008. Hostspecific toxins: Effectors of necrotrophic pathogenicity. Cell. Microbiol. 10:1421-1428.

Ghareeb, H., Becker, A., Iven, T., Feussner, I., and Schirawski, J. 2011. Sporisorium reilianum infection changes inflorescence and branching architectures of maize. Plant Physiol. 156:2037-2052.

Greenberg, J. T. 2007. Programmed cell death in plant pathogen interactions. Annu. Rev. Plant Physiol. Plant Mol. Biol. 48:525-545.

Halisky, P. M. 1963. Head smut of sorghum, sudan grass, and corn, caused by Sphacelotheca reiliana (Kühn) Clint. Hilgardia 34:287-304.

Hanna, W. F. 1929. Studies in the physiology and cytology of Ustilago zeae and Sorosporium reilianum. Phytopathology 19:415-442.

Liu, H., Du, Y., Chu, H., Shih, C. H., Wong, Y. W., Wang, M., Chu, I. K., Tao, Y., and Lo, C. 2010. Molecular dissection of the pathogen-inducible 3-deoxyanthocyanidin biosynthesis pathway in sorghum. Plant Cell. Physiol. 51:1173-11785.

Lo, S. C., and Nicholson, R. L. 1998. Reduction of light-induced anthocyanin accumulation in inoculated sorghum mesocotyls. Implications for a compensatory role in the defense response. Plant Physiol. 116:979-989.

Lo, S.-C., De Verdier, K. and Nicholson, R. L. 1999. Accumulation of 3deoxyanthocyanidin phytoalexins and resistance to Colletotrichum sublineolum in sorghum. Physiol. Mol. Plant Pathol. 55:263-273.
Martinez, C., Roux, C., and Dargent, R. 1999. Biotrophic development of Sporisorium reilianum f. $\mathrm{sp}$. zeae in vegetative shoot apex of maize. Phytopathology. 89:247-253.

Martinez, C., Roux, C., Jauneau, A., Dargent, R. 2002. The biological cycle of Sporisorium reilianum f.sp. zeae: An overview using microscopy. Mycologia 94:505-514.

Matyac, C. A., and Kommedahl, T. 1985a. Factors affecting the development of head smut caused by Sphacelotheca reiliana on corn. Phytopathology 75:577-581.

Matyac, C. A., and Kommedahl, T. 1985b. Occurrence of chlorotic spots on corn seedlings infected with Sphacelotheca reiliana and their use in evaluation of head smut. Plant Dis. 69:251-254.

Mehrabi, R., Bahkali, A. H., Abd-Elsalam, K. A., Moslem, M., Ben M'barek, S., Gohari, A. M., Jashni, M. K., Stergiopoulos, I., Kema, G. H., and de Wit, P. J. 2011. Horizontal gene and chromosome transfer in plant pathogenic fungi affecting host range. FEMS (Fed. Eur. Microbiol. Soc.) Microbiol. Rev. 35:542-554.

Nicholson, R. L., Kollipara, S. S., Vincent, J. R., Lyons, P. C., and CadenaGomez, G. 1987. Phytoalexin synthesis by the sorghum mesocotyl in response to infection by pathogenic and nonpathogenic fungi. Proc. Natl. Acad. Sci. U.S.A. 84:5520-5524.

Pedras, M. S. C., and Ahiahonu, P. W. K. 2005. Metabolism and detoxification of phytoalexins and analogs by phytopathogenic fungi. Phytochemistry 66:391-411.

Prom, L. K., Perumal, R., Erattaimuthu, S. R., Erpelding, J. E., Montes, N., Odvody, G. N., Greenwald, C., Jin, Z., Frederiksen, R., and Magill, C.W. 2011. Virulence and Molecular Genotyping Studies of Sporisorium reilianum Isolates in Sorghum. Plant Dis. 95:523-529.

Schirawski, J., Heinze, B., Wagenknecht, M., and Kahmann, R. 2005. Mating type loci of Sporisorium reilianum: Novel pattern with three $a$ and multiple $b$ specificities. Eukaryot. Cell 4:1317-1327.

Schirawski, J., Mannhaupt, G., Münch, K., Brefort, T., Schipper, K., Doehlemann, G., Di Stasio, M., Rössel, N., Mendoza-Mendoza, A., Pester, D., Müller, O., Winterberg, B., Meyer, E., Ghareeb, H., Wollenberg, T., Münsterkötter, M., Wong, P., Walter, M., Stukenbrock, E., Güldener, U., and Kahmann, R. 2010. Pathogenicity determinants in smut fungi revealed by genome comparison. Science. 330:15461548.

Shih, C. H., Siu, S. O., Ng, R., Wong, E., Chiu, L. C., Chu, I. K., and Lo, C. 2007. Quantitative analysis of anticancer 3-deoxyanthocyanidins in infected sorghum seedlings. J. Agric. Food Chem. 55:254-259.

Snyder, B. A., and Nicholson, R. L. 1990. Synthesis of phytoalexins in sorghum as a site-specific response to fungal ingress. Science. 248:1637-1639.

Stromberg, E. L., Stienstra, W. C., Kommedahl, T., Matyac, C. A., Windels, C. E., and Geadelmann, J. L. 1984. Smut expression and resistance of corn to Sphacelotheca reiliana in Minnesota. Plant Dis. 68:880-884.

Tanaka, A., Shiotani, H., Yamamoto, M., and Tsuge, T. 1999. Insertional mutagenesis and cloning of the genes required for biosynthesis of the host-specific AK-toxin in the Japanese pear pathotype of Alternaria alternata. Mol. Plant-Microbe Interact. 12:691-702.

Touraud, G., Bill, L., and Piollat, M. T. 1997. Phyllodied panicles caused by head smut of maize and vegetative multiplication of maize. Plant Cell 50:19-26.

VanEtten, H. D., Matthews, D. E., and Matthews, P. S. 1989. Phytoalexin detoxification: Importance for pathogenicity and practical implications. Annu. Rev. Phytopathol. 27:143-164. 